\title{
Effect of nonsurgical periodontal therapy on crevicular fluid and serum glutathione peroxidase levels
}

\author{
Swati Pradeep Patel, Nishanth S. Rao and A.R. Pradeep* \\ Department of Periodontics, GDCRI, Bangalore, India
}

\begin{abstract}
Background: Plasma glutathione peroxidase (eGPx) is an important selenium containing antioxidant in human defense against oxidative stress. While crevicular fluid (GCF) eGPx levels and its association with periodontal disease is well documented, there is no data on correlation of GCF and serum eGPx levels in chronic periodontitis. Hence this study was undertaken to further probe into the role of oxidative stress in periodontal diseases and effect of nonsurgical periodontal therapy (NSPT) by correlating GCF and serum levels of eGPx.

Materials and methods: Thirty subjects (16-Males and 14-Females; age: 30-38 years) participated in the study. The subjects were divided, based on gingival index, probing pocket depth and clinical attachment level into: Healthy (group-1, $n=10$ ), Gingivitis (group-2, $n=10$ ) and Periodontitis (group-3, $n=10$ ). Chronic periodontitis patients after NSPT constituted group 4. GCF and serum samples collected from each subject were quantified for eGPx levels using Enzyme linked Immunosorbent Assay.

Results: The mean eGPx concentrations increased from health $(14.01 \mathrm{ng} / \mu 1$ and $78.26 \mathrm{ng} / \mathrm{ml})$ to gingivitis $(22.86 \mathrm{ng} / \mu 1$ and $90.44 \mathrm{ng} / \mathrm{ml}$ ) and then to periodontitis $(29.89 \mathrm{ng} / \mu \mathrm{l}$ and $103.43 \mathrm{ng} / \mathrm{ml})$, in GCF and serum respectively. After NSPT, there was statistically significant reduction in eGPx concentration in GCF and serum $(19.41 \mathrm{ng} / \mu \mathrm{l}$ and $85.21 \mathrm{ng} / \mathrm{ml})$. Further, all the GCF eGPx values showed a positive correlation to that of serum eGPx level.

Conclusion: Thus, increased eGPx concentration in GCF can be considered as an indicator of local increase in oxidative stress. While, increase in serum eGPx levels indicates that periodontal disease can also lead to increased oxidative stress at the systemic level.
\end{abstract}

Keywords: Chronic periodontitis, glutathione peroxidase, gingival crevicular fluid, serum, dental prophylaxis, oxidative stress

\section{Introduction}

Oxidative stress refers to the overproduction of free radicals/oxidants and/or an insufficiency of antioxidant/ radical-detoxifying systems that results in deleterious modification of biological macromolecules. Oxidative stress continues to be linked to an ever-increasing number of dysfunctional states in vivo, of which atherosclerosis, neurodegenerative diseases, cancer, inflammation, and the normal aging process are only a few exam-

* Corresponding author: Department of Periodontics, Government Dental College and Research Institute, Bangalore-560002, Karnataka, India. Tel.: +91 9845081190; E-mail: periodontics_gdc@ rediffmail.com. ples. The deleterious effects of oxidative stress that contribute to disease most frequently manifest as chemical modification of proteins, nucleic acids, and lipids [1].

Periodontitis is a chronic inflammatory disease caused by oral bacterial infection [2]. When stimulated by bacterial pathogens, host cells (e.g., polymorphonuclear leukocytes) release reactive oxygen species (ROS) as a part of the immune response [3]. Excessive production of ROS in polymorphonuclear leukocytes is one of the pathologic features in the periodontal lesion [4], and it leads to damage of the periodontal tissue by oxidizing DNA, lipids, and proteins [5].

Protection against such species is provided by antioxidants (AO's). In healthy organisms, the balance is maintained among oxidants and AO's but under patho- 
logic conditions, the balance may be tilted towards the oxidative side causing "oxidative stress". Oxidative stress is implicated in numerous human diseases, such as arthritis, adult respiratory distress syndrome, heart disease, stroke and many others including periodontal diseases [5]. But the defense mechanisms against oxidative stress in periodontal diseases have received little attention.

Glutathione peroxidases (GPX) are important selenium containing antioxidants in human defense against oxidative stress. Plasma glutathione peroxidase (eG$\mathrm{Px}$ ) is unique among the members of GPx family as the only extracellular isoform [6]. It is a glycosylated tetramer of $23-\mathrm{kDa}$ subunits that catalyses the reduction of hydrogen peroxide $\left(\mathrm{H}_{2} \mathrm{O}_{2}\right)$ and various hydroperoxidase using glutathione as reducing agent [6]. In humans, eGPx is produced mainly in proximal tubules of the kidney nephrons [7]. The gene expression of eGPx is reported to be upregulated by $\mathrm{H}_{2} \mathrm{O}_{2}$ and other ROS [8]. In a recent study it is demonstrated that human airway epithelial cells and alveolar macrophages of smoking individual showed increased expression of eGPx mRNA [9]. The same investigators in a later study found that patients with asthma have higher levels of eGPx mRNA in their airway epithelia than do healthy controls, thus postulating a possible local production of eGPx in lung alveoli [10]. Increased plasma eGPx level has been reported as an indirect indicator of oxidative stress in patients with inflammatory bowel disease [11]. Recently, low levels of GPx activity and elevated levels of myeloperoxidase were shown to be an independent risk factor for cardiovascular events in patients with coronary artery disease and in patients presenting with chest pain [12]. In CKD patients, eGPx activity has always been shown to be reduced by $34-52 \%$ as compared with healthy. Several authors have indicated a gradual decrease in the activity with advancing stage of the disease [13]. A progressive decline in eGPx activity is linked with the fact that this enzyme is primarily synthesized in the kidney [7] and as generally believed the progressing damage of this organ is reflected in diminished enzyme activity.

In our recent study, the eGPx levels in gingival crevicular fluid (GCF) in periodontal health, disease and after non surgical periodontal therapy [(NSPT) or scaling and root planing (SRP)] were estimated. We found that eGPx concentration in GCF increases proportionally with the severity of periodontal disease. Further, the treatment aimed at arresting periodontal disease resulted in statistically significant reduction in the levels of GCF eGPx proportionally [14]. However till date no study has correlated the GCF and serum levels of eGPX in periodontal health and disease.

Thus, in view of the aforementioned findings, this clinicobiochemical study was undertaken to further probe into the role of oxidative stress in periodontal diseases by determining the effect of nonsurgical periodontal therapy on crevicular fluid and serum glutathione peroxidase levels in subjects with clinically healthy periodontium, in patients with gingivitis, chronic periodontitis, and after NSPT of periodontitis subjects.

\section{Materials and methods}

The study was carried out from February 2008 to June 2008. Thirty subjects who were age- (3038 years) and sex- (16 males and 14 females) matched, attending the outpatient section, Department of Periodontics, Government Dental College and Research Institute, Bangalore, India, were selected for the study. Written informed consent was obtained from those who agreed to participate voluntarily and the ethical clearance was obtained from the institutions ethical committee and review board. The study was performed in full accordance with ethical principles including the World Medical Association Declaration of Helsinki. Exclusion criteria included: history of smoking, diseases such as chronic obstructive pulmonary disease (COPD), asthma, bronchitis, rheumatoid arthritis, renal disorders, diabetes, tumours and the subjects who have received anti-inflammatory, antibiotics and antioxidants in the previous 6 months. Each subject underwent a full-mouth periodontal probing and charting, along with periapical radiographs using the longcone technique. Radiographic bone loss was recorded dichotomously (presence or absence) to differentiate chronic periodontitis patients from other groups. Further, no delineation was attempted within the chronic periodontitis group based on the extent of alveolar bone loss. Based on the gingival index (GI) [15], pocket probing depth (PPD), clinical attachment loss (CAL) and radiograph evidence of bone loss subjects were categorized into three groups. Group 1 (healthy) consisted of 10 subjects with clinically healthy periodontium, with GI $=0$, with $\mathrm{CAL}=0 \mathrm{~mm}$ and PPD $\leqslant$ $3 \mathrm{~mm}$. Group 2 (gingivitis) consisted of 10 subjects who showed clinical signs of gingival inflammation, GI $>1$, without any attachment loss, PPD $\leqslant 3 \mathrm{~mm}$. Group 3 (chronic periodontitis) consisted of 10 subjects who had signs of clinical inflammation, GI $>1$ and CAL 
$\geqslant 1 \mathrm{~mm}$ in $30 \%$ of sites with radiographic evidence of bone loss and PPD $\geqslant 4 \mathrm{~mm}$ in $30 \%$ of sites. Patients with chronic periodontitis (group 3) were treated with a non-surgical approach, i.e., NSPT and GCF samples collected from the same sites $6-8$ weeks after treatment to constitute group 4 (after treatment group).

\section{Site selection and GCF collection}

All the clinical and radiological examinations, group allocation and sampling site selection were performed by one examiner (ARP) and the samples were collected on the subsequent day by second examiner (SP). This was done to prevent the contamination of GCF with blood associated with probing of inflamed sites. Only one site per subject was selected as a sampling site. In healthy group, to ensure adequate volume, GCF was pooled from multiple sites with no inflammatory signs. In gingivitis patients, site with highest clinical signs of inflammation i.e., redness, bleeding on probing and oedema in the absence of CAL was selected. In chronic periodontitis patients, sites were identified using a University of North Carolina (UNC)-15 periodontal probe (Hu-Friedy, Inc. Chicago, Illinois (IL), USA) and the site showing highest clinical signs of inflammation and highest CAL along with radiographic confirmation of bone loss was selected for sampling. On the subsequent day, after gently drying the area with a blast of air, supragingival plaque was removed without touching the marginal gingiva. The area was isolated using cotton rolls to prevent the saliva contamination and GCF was collected by placing the microcapillary pipettes at the entrance of the gingival sulcus, gently touching the marginal gingiva. From each test site, a standardized volume of $1 \mu \mathrm{l}$ was collected using the calibration on white colour-coded $1-5 \mu$ l calibrated volumetric microcapillary pipettes (Sigma-Aldrich, St. Louis, MO, USA). Each sample collection was allotted a maximum of $10 \mathrm{~min}$ (due to slow rate of GCF production in certain healthy subjects) and some test sites in healthy group, which did not express any volume of GCF within the allotted time, were excluded from the study. Further, micropipettes, which were suspected to be contaminated with blood and saliva, were excluded from the study. The GCF collected was immediately transferred to airtight plastic vial and stored at $-70^{\circ} \mathrm{C}$ till the time of assay.

\section{Collection of serum}

Two millilitre of blood was collected from the antecubital fossa by venipuncture using a 20 -gauge needle with $2 \mathrm{ml}$ syringes and immediately transferred to the laboratory. The blood sample was allowed to clot at room temperature and, after one hour, serum was extracted from blood by centrifuging at $3000 \mathrm{~g}$ for $5 \mathrm{~min}$. The extracted serum was immediately transferred to a plastic vial and stored at $-70^{\circ} \mathrm{C}$ till the time of assay.

\section{5. eGPx assay}

The concentration of eGPx was determined by enzyme linked immunoassay kit (ELISA) (Plasma glutathione peroxidase Assay Kit, Calbiochem, USA) for the quantitative determination of eGPx in biologic fluids, as instructed by the manufacturer. Appropriately diluted samples were incubated in the wells of a divided microplate that have been coated with a polyclonal antibody specific for human eGPx. The eGPx was detected using a biotinylated polyclonal antibody to eGPx. The assay procedure, based on biotin-streptavidin coupling, used covalently linked streptavidin and alkaline phosphatase to measure the amount of eGPx in each sample by adding the alkaline phosphatase substrate p-nitrophenylphosphate (pNPP). Absorbance of each well was read on ELISA reader (Molecular Dynamics, Sunnyvale, USA) using $405 \mathrm{~nm}$ as primary wavelength. The concentration of eGPx in the tested samples was evaluated using the standard curve plotted using the absorbance value obtained for the standards provided with the kit.

\section{Statistical analysis}

All data were analyzed using a software program (Systat, version 11, and Sigmastat, Systat software, Point Richmond, CA). Group comparisons for variables were performed by ANOVA. Further, multiple comparisons using the scheff test was carried out to explore which pair or pairs differed. The Correlation between GCF and serum eGPx with clinical periodontal parameters was analyzed using Spearman's rank correlation coefficient. $P$-value $\leqslant 0.05$ was considered to indicate statistical significance. The sample size based on power analysis was estimated as 10 subjects in each group to achieve $80 \%$ power to detect a difference of 0.5 between the null hypothesis and the alternative mean.

\section{Results}

All the samples, in each group tested positive for the presence of eGPx. The mean eGPx concentrations in 
Table 1

Descriptive StatisticsShowing the gender distribution, age,GI, PPD, CAL, GCF and Serum eGPx concentration of the study population

\begin{tabular}{lcccc}
\hline & $\begin{array}{c}\text { Group 1 } \\
(n=10)\end{array}$ & $\begin{array}{c}\text { Group 2 } \\
(n=10)\end{array}$ & $\begin{array}{c}\text { Group 3 } \\
(n=10)\end{array}$ & $\begin{array}{c}\text { Group 4 } \\
(n=10)\end{array}$ \\
\hline Sex (M:F) & $5: 5$ & $6: 4$ & $5: 5$ & $5: 5$ \\
Age (years) & $35.10 \pm 2.02$ & $34.20 \pm 2.53$ & $35.10 \pm 2.51$ & $35.10 \pm 2.51$ \\
GI & 0.287 & 2.16 & 2.25 & 0.315 \\
PPD $(\mathrm{mm})$ & 1.3 & 2.2 & 6.1 & 3.6 \\
CAL $(\mathrm{mm})$ & 0 & 0 & 4.1 & 1.9 \\
GCF eGPx $(\mathrm{ng} / \mu \mathrm{l})$ & $14.01 \pm 2.8646$ & $22.87 \pm 3.2658$ & $29.89 \pm 4.0346$ & $19.41 \pm 2.9069$ \\
Serum eGPx $(\mathrm{ng} / \mathrm{ml})$ & $78.25 \pm 4.6494$ & $90.44 \pm 4.5629$ & $103.43 \pm 7.1637$ & $85.22 \pm 6.8182$ \\
\hline
\end{tabular}

Table 2

Results of ANOVA comparing the mean eGPx concentrations in GCF and Serum between groups 1,2 and 3

\begin{tabular}{lccccccc}
\hline Study groups & \multicolumn{3}{c}{ GCF eGPx } & & \multicolumn{3}{c}{ Serum eGPx } \\
\cline { 2 - 3 } \cline { 6 - 8 } & No of samples & $\mathrm{F}$ & P-value & & No of samples & $\mathrm{F}$ & P-value \\
\hline Group 1 & 10 & & & & 10 & \\
Group 2 & 10 & 40.560 & $<0.01^{*}$ & & 10 & 32.308 & $0.01^{*}$ \\
Group 3 & 10 & & & & 10 & & \\
\hline
\end{tabular}

${ }^{*}$ The mean difference is significant at $p<0.05$.

Table 3

Pair-Wise comparison using scheff test for GCF and serum eGPx for groups 1, 2 and 3

\begin{tabular}{lcllcr}
\hline Study groups & \multicolumn{2}{c}{ GCF } & & \multicolumn{2}{c}{ Serum } \\
\cline { 2 - 3 } \cline { 5 - 6 } \cline { 5 - 6 } & Mean difference & P-value & & Mean difference & P-value \\
\hline Group 1 Vs Group 2 & -8.85 & $<0.001^{*}$ & & 12.19 & $0.001^{*}$ \\
Group 1 Vs Group 3 & 15.87 & $<0.001^{*}$ & & 25.18 & $<0.001^{*}$ \\
Group 2 Vs Group 3 & -7.02 & $<0.001^{*}$ & & 12.99 & $<0.001^{*}$ \\
\hline
\end{tabular}

${ }^{*}$ The mean difference is significant at $p<0.05$.

GCF and serum were observed to be the highest in group 3 i.e. $29.89 \mathrm{ng} / \mu \mathrm{l}(29890 \mathrm{ng} / \mathrm{ml})$ and $103.43 \mathrm{ng} / \mathrm{ml}$ respectively and lowest in group 1 i.e. $14.01 \mathrm{ng} / \mu \mathrm{l}$ (14010 ng/ml) and $78.26 \mathrm{ng} / \mathrm{ml}$ respectively (Table 1).

To test the hypothesis of equality of means among the groups ANOVA was carried out, which indicated that the means differed significantly (Table 2).

Further multiple comparisons using scheff test showed that when groups 1 and 2, 1 and 3 and 2 and 3 were compared, both in GCF and serum, the difference were statistically significant $(p<0.05)$ (Table 3$)$.

When group 4 (after treatment group) and group 3 were compared using paired ' $t$ ' test, the difference in the concentrations of eGPx was statistically significant in both serum and GCF suggesting that after scaling and root planning, eGPx levels decreased considerably both in GCF (from $29.89 \mathrm{ng} / \mu \mathrm{l}$ to $19.41 \mathrm{ng} / \mu \mathrm{l}$ ) and proportionally in serum (from $103.43 \mathrm{ng} / \mathrm{ml}$ to 85.21 ng/ml) (Table 4).

Pearson Correlation Coefficient test showed a positive but non-significant correlation between eGPx concentration in GCF and serum and the clinical parameters in all the groups except in group 4. A signifi- cant positive correlation between eGPx concentration in GCF and serum was found in group 3 and 4 (Table 5).

\section{Discussion}

eGPx is an important extracellular antioxidant in human defence against oxidative stress, produced mainly in kidney. It is an selenium containing enzyme that detoxifies hydrogen peroxide and various hydroperoxides using glutathione as a reducing agent [6].

The findings in our previous research were consistent with the results of Wei et al. and it was found that in periodontitis patients, both total amount and concentration of eGPx were higher in GCF as compared to the levels in healthy patients $[14,16]$, and the levels of eGPx from the gingivitis sites of periodontitis patients were less that the levels in periodontitis sites but were higher than those of healthy patients. However, Huang et al. [17] found that within periodontitis subjects, glutathione peroxidase levels correlated negatively with pocket depth and attachment loss and increased posttherapy. Till today, there are no studies which have 
Table 4

Paired 't' test to compare eGPx concentrations in serum and GCF in group 3 and group 4

\begin{tabular}{lcrrr}
\hline & \multicolumn{2}{c}{ Paired Differences } & \multirow{2}{*}{$\mathrm{t}$} & 'P' value \\
\cline { 2 - 3 } & Mean & SD & & \\
\hline Group 3-Serum - Group 4-Serum & 18.2200 & 3.0723 & 18.754 & $0.001^{*}$ \\
Group 3-GCF - Group 4-GCF & 10.4750 & 3.8137 & 8.686 & $0.001^{*}$ \\
\hline
\end{tabular}

* The mean difference is significant at $p<0.05$.

Table 5

Pearson Correlation Coefficient test comparing GCF eGPx, serum eGPx, GI, PPD and CAL

\begin{tabular}{|c|c|c|c|c|c|c|c|}
\hline Groups & $\begin{array}{c}\text { GCF and } \\
\text { Serum }\end{array}$ & $\begin{array}{c}\text { GCF and } \\
\text { GI }\end{array}$ & $\begin{array}{c}\text { GCF and } \\
\text { PPD }\end{array}$ & $\begin{array}{c}\text { GCF and } \\
\text { CAL }\end{array}$ & $\begin{array}{c}\text { Serum and } \\
\text { GI }\end{array}$ & $\begin{array}{c}\text { Serum and } \\
\text { PPD }\end{array}$ & $\begin{array}{c}\text { Serum and } \\
\text { CAL }\end{array}$ \\
\hline Group 1 & 0.371 & 0.070 & $0.762^{*}$ & - & -0.005 & 0.462 & - \\
\hline Group 2 & 0.252 & 0.402 & 0.194 & - & $0.657^{*}$ & 0.160 & - \\
\hline Group 3 & $0.799^{* *}$ & 0.190 & 0.285 & 0.072 & 0.036 & 0.327 & 0.179 \\
\hline Group 4 & $0.690^{*}$ & -0.181 & -0.378 & -0.448 & 0.325 & -0.265 & -0.316 \\
\hline
\end{tabular}

${ }^{*}$ Correlation is significant at the 0.05 level (2-tailed).

** Correlation is significant at the 0.01 level (2-tailed).

investigated the eGPx concentration in GCF and correlated them to that of serum eGPx concentrations in periodontal health, disease and after treatment of periodontal disease. The present study is thus the first study designed to investigate the concentrations of eGPx in GCF and serum in periodontal health and disease and also to assess the role of periodontal therapy on eGPx concentration.

In the present study the influence of age and gender of the subjects on the eGPx concentration was minimised/ nullified by selecting the subjects within the narrow age group of 30-38 years and including the equal number of males and female in each group. Further, this study comprised of four groups (healthy, gingivitis, chronic periodontitis and chronic periodontitis after treatment) as compared to the previous study by Wei et al. [16] where in the effect of treatment on eGPx levels was not evaluated. Also, in their study the gingivitis and periodontitis sites were selected from the same group of chronic periodontitis patients where as we included different patients in all the groups. Having four groups in our study helped us better to evaluate the role of eGPx in different stages of periodontal and the effect of periodontal therapy on eGPx concentrations.

In the present study, the use of commercially available, sensitive ELISA kit to quatify eGPx from selected sites allowed us to avoid pooling of GCF samples from multiple sites or subjects. Also, GCF was collected using microcapillary pipettes to avoid non-specific attachment of the analyte to filter paper fibers ensuing in false reduction in the detectable eGPx levels that in turn can underestimate the correlation of eGPx levels to disease severity/ progression.
The results of the present study indicated that concentration of eGPx in GCF increased progressively from healthy $(14.01 \mathrm{ng} / \mu \mathrm{l}$ i.e. $14010 \mathrm{ng} / \mathrm{ml})$ to periodontitis sites $(29.89 \mathrm{ng} / \mu \mathrm{l}$ i.e. $29890 \mathrm{ng} / \mathrm{ml})$, while in gingivitis the mean concentration of eGPx $(22.87 \mathrm{ng} / \mu \mathrm{l}$ i.e. $22890 \mathrm{ng} / \mathrm{ml}$ ) fell between the above two groups. The results of our study was in accordance to Wei et al. [16] who also reported a similar increase in the concentration of eGPx from health to disease.

The findings seem to confirm several findings in the medical literature about oxidant-antioxidant balance. Mates et al. [8] reported that there was upregulation of eGPx gene expression by $\mathrm{H} 2 \mathrm{O} 2$ and other ROS. Similarly Comhair et al. [9] showed that human airway epithelial cells and alveolar macrophages of smoking individuals showed increased expressions of eGPx mRNA. In the present study increased eGPx level in GCF from inflamed gingiva may indicate the increase ROS generation at the diseased site. This increase in ROS generation may have led to the occurrence of oxidative stress, which in turn caused an increased need of eGPx production to establish the ROS-AO balance to protect the tissues.

Further in our study, the mean eGPx levels in serum were observed to be the highest in chronic periodontitis group (103.44 ng/ml) and lowest for the healthy group $(78.26 \mathrm{ng} / \mathrm{ml})$ with gingivitis group showing intermediate values $(90.45 \mathrm{ng} / \mathrm{ml})$. Thus the mean concentration of eGPx increased progressively from health to gingivitis and periodontitis both in GCF and proportionally in serum.

Increased plasma eGPx has been reported as an indirect marker of oxidative stress in patients with in- 
flammatory bowel disease [11]. Similarly, increase in GCF and serum eGPx can be considered as a marker of oxidative stress caused by periodontal infection. Oxidative stress has been implicated in the development of atherosclerosis and vascular tissue injury. Both platelet activation and lipid peroxidation are known to play major role in ischemic heart disease [18].

The chronic periodontitis subjects were treated by non-surgical periodontal therapy and strict oral hygiene measures were instituted. The mean concentration of eGPX in GCF and serum in chronic periodontitis group reduced from $29.89 \mathrm{ng} / \mu \mathrm{l}(29890 \mathrm{ng} / \mathrm{ml})$ and $103.44 \mathrm{ng} / \mathrm{ml}$ to an after treatment level of $19.42 \mathrm{ng} / \mu \mathrm{l}$ $(19420 \mathrm{ng} / \mathrm{ml})$ and $85.22 \mathrm{ng} / \mathrm{ml}$ respectively, which were statistically significant.

Data from GCF support the conclusion that local antioxidant scavenging defenses are compromised in periodontitis, but whether this represents a predisposition to disease or results from the inflammatory lesion, requires longitudinal studies of periodontal therapy to be performed [5].

The significant increase in serum eGPx concentration with the progressing periodontal disease can be possibly because of spillover from the diseased periodontal tissues or could be because of increased production of eGPx by kidney proximal tubules in response to systemic oxidative stress caused by periodontal disease. This highlights the possible systemic oxidative stress caused by periodontal disease, which could be a missing link between the periodontal and systemic health. This finding further signifies the need of controlling the periodontal inflammation to reduce the systemic oxidative stress.

Further, when the concentration of eGPx in GCF was compared to that of serum, the concentration of eGPx in GCF was significantly higher that corresponding serum concentration in all the groups. It seems logical that serum will provide the basic antioxidant profile of crevicular fluid, as there is constant flow of fluid from the blood into the gingival crevice [19]. However, the finding that the concentrations of eGPx in GCF are significantly greater than those in paired samples of serum suggests a possible local eGPx synthesis and/or storage within the periodontium. The likelihood of local production by the recruiting inflammatory cells cannot be overlooked.

The eGPx present in serum of chronic periodontitis patients as a result of local oxidative stress may also in turn offer a certain degree of protection against systemic oxidative stress. Considering this role we can consider evaluating its therapeutic application in future studies regarding the use of antioxidants and antioxidant enzymes not only in the management of chronic periodontitis but also for systemic diseases as well.

Thus our study shows that eGPx concentration in GCF and serum increases proportionally with the progression of periodontal disease. Further, the treatment aimed at arresting periodontal disease progression resulted in statistically significant reduction in the levels of eGPx both in serum and GCF proportionally. Overall, the results point towards the role of oxidative stress in the periodontal diseases, and pave the way for future exploration of the role of antioxidants in the management of periodontal diseases.

Because the results of ELISA only gave us information about the concentration of eGPx, the enzymatic activity was unknown. Further investigations are needed to determine the enzymatic activities of eGPx and elucidate the interrelationship between them and periodontal diseases.

\section{Conclusion}

In conclusion, within the limitations of the present study, it can be postulated that with increase in the amount of periodontal destruction there is substantial increase in the concentration of eGPx both in GCF and serum. Also, treatment of periodontal disease leads to a proportional reduction in GCF and serum levels of eGPx.

Thus, eGPx can be regarded as a marker of oxidative stress in periodontal diseases. Furthermore, the finding that the concentrations of eGPx in GCF are significantly greater than those in paired samples of serum suggests a possible local eGPx synthesis and/or storage within the periodontium. The likelihood of local production by the recruiting inflammatory cells cannot be overlooked.

However further longitudinal studies with larger sample size and long term follow-up are needed to validate eGPx as marker of oxidative stress as well as its possible therapeutic applications in periodontal health and disease.

\section{Acknowledgement}

The authors acknowledge Mr. Jaganatha SP, (Honorary Statistician, Bangalore, India) for helping us prepare the statistics of the study, and Mr. Ventakswamy (Technician, Dept. of Microbiology, Kempegowda Institute of Medical Sciences, Bangalore) for helping us running the ELISA. 


\section{Conflict of interest and source of funding}

1. The authors report no conflict of interests.

2. It is a self funded research

\section{References}

[1] J.P. Fessel and L. Jackson Roberts II, Isofurans: Novel products of lipid peroxidation that define the occurrence of oxidant injury in settings of elevated oxygen tension, Antioxid Redox Signal 7 (2005), 202-209.

[2] R.C. Page and K. Kornman, The pathogenesis of human periodontitis: An introduction, Periodontol 200014 (1997), 9-11.

[3] D.V. Sculley, Langley-Evans SC. Salivary antioxidants and periodontal disease status, Proc Nutr Soc 61 (2002), 137-143.

[4] D. Ekuni, T. Tomofuji, N. Tamaki et al., Mechanical stimulation of gingiva reduces plasma $8-\mathrm{OHdG}$ level in rat periodontitis, Arch Oral Biol 53 (2008), 324-329.

[5] I.L. Chapple and J.B. Matthews, The role of reactive oxygen and antioxidant species in periodontal tissue destruction, Periodontol 200043 (2007), 160-232.

[6] S. Yoshimura, H. Suemizu, Y. Taniguchi, K. Arimori, N. Kawabe and T. Moriuchi, The human plasma glutathione peroxidase-encoding gene: organization, sequence and localization to chromosome 5q32, Gene 145 (1994), 293-297.

[7] N. Avissar, D.B. Ornt, Y. Yagil, S. Horowitz, R.H. Watkins, E.A. Kerl et al., Human kidney proximal tubules are the main source of plasma glutathione peroxidase, Am J Physiol 266 (1994), C367-C375.

[8] J.M. Mates, C. Perez-Gomez and I. Nunez de Castro, Antioxidant enzymes and human diseases, Clin Biochem 32 (1999), 595-603.

[9] S.A.A. Comhair, M.J. Thomassen and S.C. Erzurum, Differential induction of extracellular glutathione peroxidase and nitric oxide synthase 2 in airways of healthy individuals exposed to $100 \%$ O2 or cigarette smoke, Am J Respir Cell Mol Biol $\mathbf{2 3}$ (2000), 350-354.

[10] S.A.A. Comhair, P.R. Bhathena, C. Farver, F.B.J.M. Thunnissen and S.C. Erzurum, Extracellular glutathione peroxidase induction in asthamatic lungs: evidence for redox regulation of expression in human airway epithelial cells, FASEB $J \mathbf{1 5}$ (2001), 70-78.

[11] A. Tüzün, A. Erdil, V. Ihal, A. Aydin, S. Bagci, Z. Yesilova et al., Oxidative stress and antioxidant capacity in patients with inflammatory bowel disease, Clin Biochem 35 (2002), $569-572$.

[12] S. Blankenberg, H.J. Rupprecht and C. Bickel, Glutathione peroxidase 1 activity and cardiovascular events in patients with coronary artery disease, $N$ Engl J Med $\mathbf{3 4 9}$ (2003), 1605-1613.

[13] I. Ceballos-Picot, V. Witko-Sarsat and M. Merad-Boudia, Glutathione antioxidant system as a marker of oxidative stress in chronic renal failure, Free Rad Biol Med 21 (1996), 845-853.

[14] S.P. Patel, A.R. Pradeep and S. Chowdhry, Crevicular fluid levels of plasma glutathione peroxidase (eGPx) in periodontal health and disease, Arch Oral Biol 54 (2009), 543-548.

[15] H. Loe and J. Silness, Periodontal disease in pregnancy. I. Prevalence and severity, Acta Odontol Scand 21 (1963), 533.

[16] P.F. Wei, K.Y. Ho, Y.P. Ho, Y.M. Wu, Y.H. Yang and C.C. Tsai, The investigation of glutathione peroxidase, lactoferrin, myeloperoxidase and interleukin-1beta in gingival crevicular fluid: implications for oxidative stress in human periodontal diseases, J Periodontal Res 39 (2004), 287-293.

[17] P. Huang, T. Su and H. Wang, The relationship between GPx activity in gingival fluid and clinical parameters of adult periodontitis, Ниа Xi Кои Qiang Yi Xие Za Zhi 18 (2000), 106108.

[18] M. Garelnabi, V. Gupta, V. Mallika and J. Bhattacharjee, Platelets oxidative stress in Indian patients with ischemic heart disease, J Clin Lab Anal 24 (2010), 49-54.

[19] Y. Kowashi, F. Jaccard and G. Cimasoni, Sulcular polymorphonuclear leucocytes and gingival exudate during experimental gingivitis in man, J Periodontal Res 15 (1980), 151-158. 


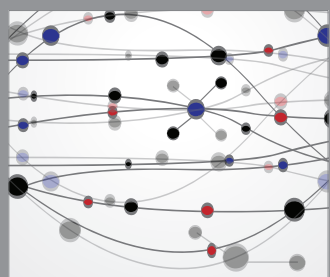

The Scientific World Journal
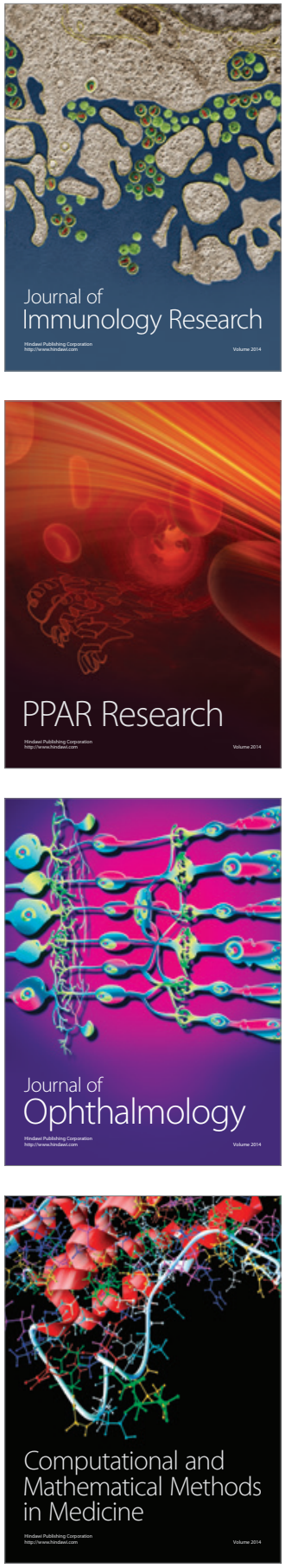

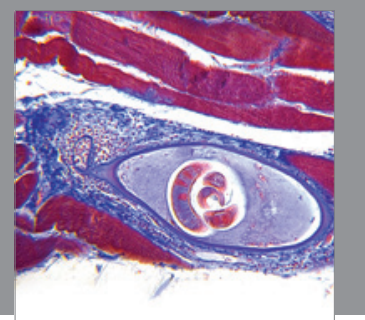

Gastroenterology

Research and Practice
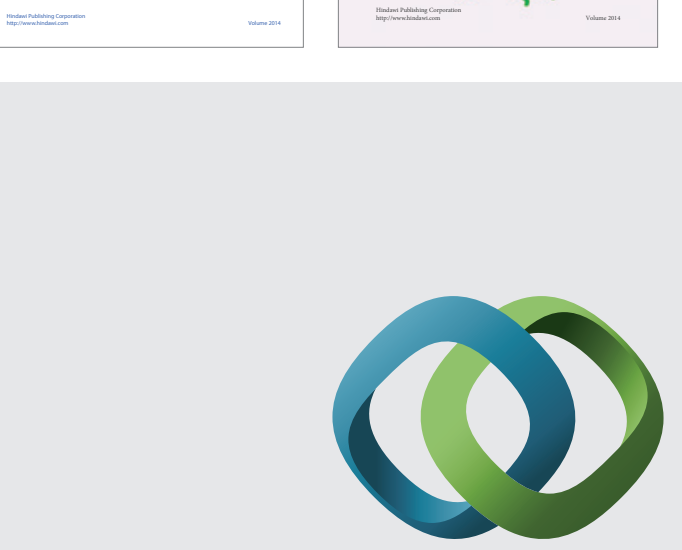

\section{Hindawi}

Submit your manuscripts at

http://www.hindawi.com
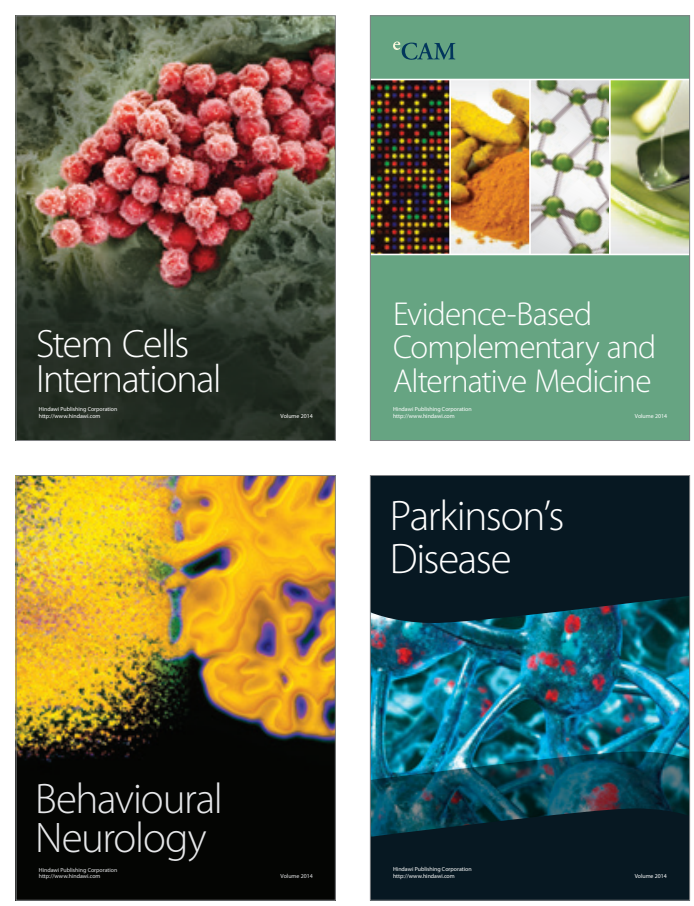

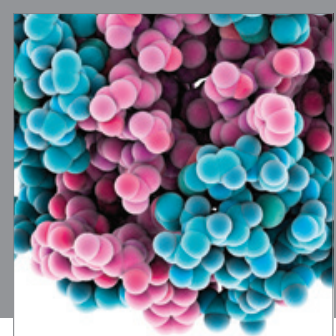

Journal of
Diabetes Research

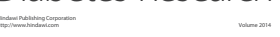

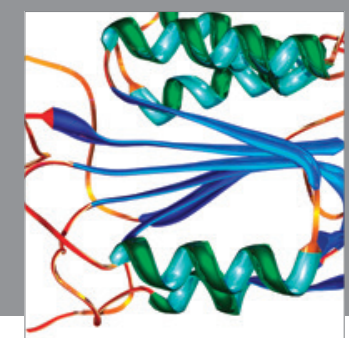

Disease Markers
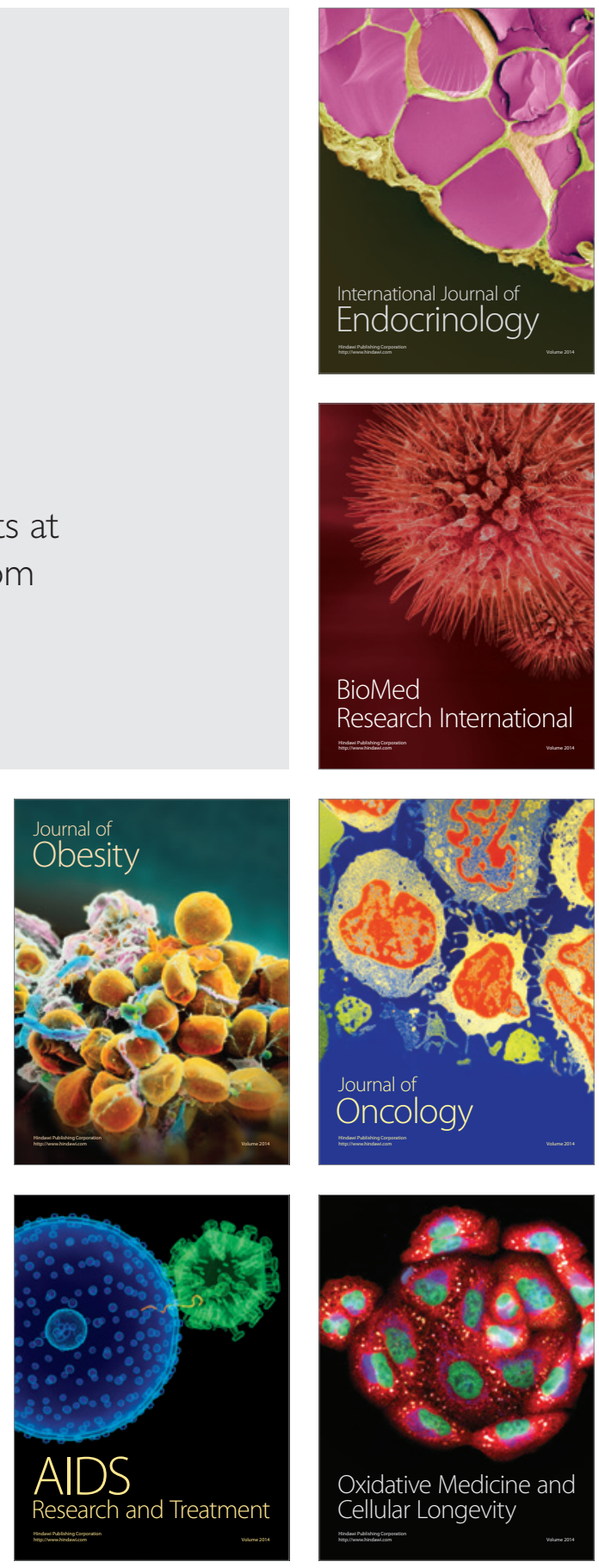